\title{
PHONON IMAGES OF CRYSTALS FOR DIFFERENT SOURCES
}

\author{
Cz. Jasiukiewicz and T. Paszkiewicz \\ Institute of Theoretical Plysics, University of Wrocław \\ Pl. M. Borna 36, 50-204 Wrocław, Poland
}

\begin{abstract}
The focusing patterns for energy and quasi-momentum are obtained for different types of phonon sources. We considered point and extended (Gaussian) sources and sources of monochromatic and Planckian phonons.

PACS numbers: $63.20 . \mathrm{Kl}, 66.70 .+f$
\end{abstract}

\section{Introduction}

In our previous papers, we have considered the energy and quasi-momentum focusing patterns [1] (hereafter referred to as I) and the image of crystalline GaAs obtained by the phonon-drag effect in $\mathrm{GaAs}-\mathrm{AlGaAs}$ heterostructures [2]. All of these patterns were obtained for sources of pulses of monochromatic phonons. The duration time of these pulses was taken as the shortest of the characteristic times in the considered problem.

Ilowever, most experiments are performed not for pulses of monochromatic phonons but for heat pulses. Additionally, the time of duration of pulses may not be the shortest time characterizing the generation, propagation and detection of pulsed phonon beams. Therefore our approach should be generalized.

In this paper we shall show that the kinetic approach, based on the use of the Boltzmann equation, can be used for the description of experiments with the use of heat pulses.

Since mechanisms for the generation of phonon beams seem not yet properly understood we construct the source terms of the Boltzmann equation using arguments based on dimensional analysis and imposing suitable normalization conditions $[1,2,3]$.

Let us briefly describe the experiment performed by Huebener and collaborators [4, 5] and Dietsche et al. [6]. All of these experiments were performed at an ambient temperature $T$ much lower than the Debye temperature $\theta_{\mathrm{D}}$. Thus the thermal phonons were frozen out.

IIuebener et al. studied the energy focusing patterns of [001] oriented single crystalline Ge [4] and GaAs samples in the form of wafer discs. The diameters of these discs were several times greater than their thickness $(1-2 \mathrm{~mm})$. The top 
surface of the crystal to be studied was scanned with an electron beam whereas the bottom surface was in the direct contact with liquid He. The phonon wave packets generated at the top crystal surface during the scanning process propagated through specimens ballistically and were detected on the backside by a superconducting thin-film bolometer attached directly to it. The two-dimensional pattern of the phonon energy density was achieved by scanning the electron beam across the crystal surface and by recording the bolometer signal synchronously. In the case of $\mathrm{Ge}$, the influence of the thin metallic film upon the angular resolution was studied [4].

Dietzel et al. obtained quasi-momentum focusing patterns [6]. A specimen of nominally undoped GaAs was immersed in liquid He. It had the form of a wafer $\{001\}$ orientation. On the front side of it, a heterostructure with a special small bridge in the two-dimensional electron gas (2DEG) was prepared. The opposite side was covered by a metallic film in the normal (nonsuperconducting) state. Wave packets of bulk acoustic phonons were generated with a pulsed laser beam focused onto a spot. As in the experiments of Huebener et al. by scanning the laser heat source across the film surface the direction of propagation of the detected wave packets was changed. Due to the anisotropy of the medium the energy and the quasi-momentum falling onto the bridge depend on the direction of propagation. Nonequilibrium ballistic phonons are absorbed by the carriers in the 2DEG, thereby transferring their quasi-momentum in the direction of the bridge and producing a voltage in an open circuit or an electric current in a closed circuit. The 2D pattern of a suitable component of the quasi-momentum density falling onto the bridge is obtained by scanning the laser beam across the film and by recording the bridge response signal synchronously.

\section{Description of the heater and the specimen}

For simplicity suppose that one deals with a monocrystalline heater and specimen. The acoustic phonons are characterized by the direction $\widehat{\boldsymbol{q}}$ and the length $q$ of the wave vector, polarization $j(j=0,1,2)$, and frequency $\omega(Q)$, where $(Q \equiv(\widehat{Q}, q) \equiv(\widehat{\boldsymbol{q}}, q, j))$. The vector $\widehat{q}$ depends on two angles $\theta$ and $\phi$.

In the dispersionless region the phase $c$ and group velocities $v$ depend only on $\widehat{\boldsymbol{q}}$, namely

$$
c=c(\widehat{Q}), \quad v=v(\widehat{Q}),
$$

where $\omega(Q)=c(\widehat{Q}) q$, and $v_{\alpha}=\partial \omega(Q) / \partial q_{\alpha}$.

The energy spectrum of phonons is completely characterized by the dependence of the surfaces of constant energy on $q$ and $\widehat{\boldsymbol{q}}$. For dispersionless phonons all these surfaces are similar, so it is enough to consider one of them, called the slowness surface. The slowness $s(\theta, \phi)$ is defined as

$$
s(\widehat{Q}) \equiv c^{-1}(\widehat{Q}), \quad(\widehat{Q} \equiv(\theta, \phi, j)) .
$$

The slowness vector $s(\hat{Q})$ is

$$
s(\widehat{Q}) \equiv \widehat{q} s(\widehat{Q}) .
$$


The slowness surface is a polar plot of $s(\hat{Q})$. The group velocity $v(\hat{Q})$ is perpendicular to the $j$-th slowness surface at the point of its intersection with the vector $q$. It is directed towards increasing energy.

Introduce two angles $\theta_{v}^{(j)}$ and $\phi_{v}^{(j)}$, so that

$$
v_{j} \equiv\left(v_{j}, \theta_{v}^{(j)}, \phi_{v}^{(j)}\right) \text {. }
$$

The local geometry of the surfaces of constant energy is characterized by the focusing factor $\mathcal{A}(\hat{Q})$ defined as the ratio of a solid angle $\mathrm{d} \Omega_{q}$ defined by a bundle of wave vectors containing $q$ and a solid angle $\mathrm{d} \Omega_{v}$ defined by the corresponding bundle of the group velocity vectors perpendicular to the $j$-th slowness surface

$$
\mathcal{A}_{j}(\theta, \phi)=\left|\frac{\mathrm{d} \Omega_{q}}{\mathrm{~d} \Omega_{v}^{(j)}}\right| .
$$

One can relate the focusing factor to the Jacobian $J$

$$
J_{j}(\theta, \phi)=\left|\frac{\partial\left[\theta_{v}^{(j)}, \phi_{v}^{(j)}\right]}{\partial(\theta, \phi)}\right| .
$$

Namely

$$
\left|\frac{\partial\left[\theta_{v}^{(j)}, \phi_{v}^{(j)}\right]}{\partial(\theta, \phi)}\right|^{-1}=\mathcal{A}_{j}(\theta, \phi)\left|\frac{\sin \theta_{v}^{(j)}}{\sin \theta}\right| .
$$

Denote the volume of the heater by $V_{\mathrm{h}}$. If the linear dimension of the elementary cell is $a_{\mathrm{h}}$, then the heater contains $N_{\mathrm{h}}=V_{\mathrm{h}} / a_{\mathrm{h}}$ elementary cells.

One of the global characteristics of a crystalline medium is the Debye velocity $c_{D}$

$$
c_{\mathrm{D}}^{(\sigma)}=\left\langle\left[c_{\sigma}(\widehat{Q})\right]^{-3}\right\rangle_{\widehat{Q}} \equiv \frac{1}{3} \sum_{j=0}^{2} \int^{4 \pi} \frac{\mathrm{d} \hat{q}}{4 \pi} \frac{1}{c_{\sigma}^{3}(\widehat{Q})} .
$$

The index $\sigma$ enumerates the heater $(\mathrm{h})$ and the specimen (s). A related characteristic is the Debye temperature $\theta_{\mathcal{D}}^{(\sigma)}$, for example

$$
\theta_{\mathrm{D}}^{(\mathrm{h})} \simeq \frac{\hbar c_{\mathrm{D}}^{(\mathrm{h})}}{k_{\mathrm{B}} a_{\mathrm{h}}}
$$

Assume that the heater is in local equilibrium with the temperature $T_{\mathrm{h}}$ greater than the ambient temperature $T$. The suitable thermal energy $E_{\mathrm{th}}\left(T_{\mathrm{h}}\right)$ is proportional to $T_{\mathrm{h}}^{4}$

$$
E_{\mathrm{th}}\left(T_{\mathrm{h}}\right)=\frac{3 I_{3} V_{\mathrm{h}}\left(k_{\mathrm{B}} T_{\mathrm{h}}\right)^{4}}{2 \pi^{2}\left(\hbar c_{\mathrm{D}}^{(\mathrm{h})} a_{\mathrm{h}}\right)^{3}} \simeq \frac{3 I_{3}}{2 \pi^{2}} N_{\mathrm{h}}\left(\frac{T_{\mathrm{h}}}{\theta_{\mathrm{D}}^{(\mathrm{h})}}\right)^{3} k_{\mathrm{B}} T_{\mathrm{h}} .
$$

The quantity used to describe the transport of phonons across the heater-specimen interface is the transmission probability $\alpha(\mathrm{h} \rightarrow \mathrm{s})$. We shall confine ourselves to the case of diffusive scattering of phonons at the interface. A phonon with energy $\hbar \omega_{\mathrm{i}}$, wave vector $q_{\mathrm{i}}$ and polarization $j_{\mathrm{i}}$ is diffusively scattered if the resulting phonon's 
wave vector $q_{\mathrm{f}}$ and polarization $j_{\mathrm{f}}$ are completely independent of $\boldsymbol{q}_{\mathrm{i}}$ and $j_{\mathrm{i}}$. For diffusive scattering (cf. [7])

$$
\alpha(\mathrm{h} \rightarrow \mathrm{s})=\frac{\left\langle c_{\mathrm{h}}^{-2}\right\rangle_{\widehat{Q}}}{\sum_{\sigma=\mathrm{h}}^{\mathrm{s}}\left\langle{c_{\sigma}^{-2}}^{-2}(\widehat{Q})\right\rangle_{\widehat{Q}}} .
$$

From this definition it follows that

$$
\alpha(\mathrm{s} \rightarrow \mathrm{h})=1-\alpha(\mathrm{h} \rightarrow \mathrm{s})=\frac{\left\langle c_{\mathrm{s}}^{-2}\right\rangle_{\widehat{Q}}}{\sum_{\sigma=\mathrm{h}}^{\mathrm{s}}\left\langle c_{\sigma}^{-2}(\widehat{Q})\right\rangle_{\widehat{Q}}} .
$$

\section{Kinetic description of bulk phonons}

Under the conditions described in Sec. 1, the injected phonons move essentially ballistically, occasionally scattering on isotope impurity atoms. The influence of boundary scattering is negligible. Therefore, as in our previous papers [1-3], we consider the half-space filled with an anisotropic medium as a natural idealization of such conditions. In the all mentioned experiments [4-6] the source was movable and the detector (a thin superconducting film bolometer $[4,5]$ or a bridge structure in 2DEG [6]) was fixed. However, one can imagine that the source is fixed and the detector moves in the plane in which the thin film or 2DEG lies (in all the mentioned experiments it was one of the $\{001\}$ planes). Therefore, we assume that at an arbitrary point in the medium boundary (also one of the $\{001\}$ planes), say at $r=0$, there is a source generating bursts of acoustic phonons. We also assume that the directions of their wave vectors are distributed randomly over the body angle $2 \pi$. We choose the Cartesian coordinate system with the $z$-axis perpendicular to the boundary. This means that only phonons with a positive $z$-component of the group velocity travel in the direction of the detector.

The movable detector is placed at an arbitrary point $r$ of the medium (Fig. 1). The vector $r$ has length $r$ and the direction $\widehat{r} \equiv\left(\theta_{r}, \phi_{r}\right)$. The surface area of the detector is $\Delta S, \widehat{n}$ is the normal to it. In the case of a quasi-momentum detector one should specify the direction $\widehat{\eta}$ of it.

The phonon deviation function $\delta N(Q ; r, t)$

$$
\delta N(Q ; r, t)=N(Q ; r, t)-N_{0}\left(\frac{\hbar \omega}{k_{\mathrm{B}} T}\right),
$$

obeys the Boltzmann equation (BE), where $N_{0}$ is the equilibrium (Planck) distribution function and $T$ is the ambient temperature. Generally it contains a drift, source and a collision term

$$
\left[\frac{\partial}{\partial t}+v(\widehat{Q}) \cdot \nabla\right] \delta N(Q ; r, t)=\dot{\zeta}(Q ; r, t)+C[\delta N(Q ; r, t)] .
$$

We have shown that under the described conditions one can drop the collision term $C$ [2].

Suppose that the source term (production function) factorizes so that

$$
\dot{\zeta}(Q ; r, t)=A(Q) F_{1}(r) F_{2}(t) \text {. }
$$




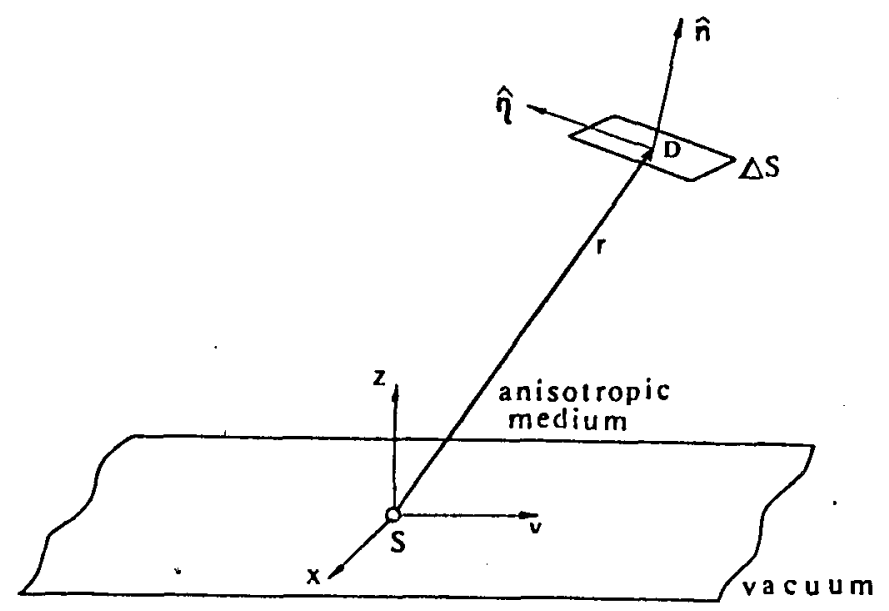

Fig. 1. Phonons are generated at the point $r=0$ of the boundary of an anisotropic medium which fills the half-space. They propagate ballistically towards a detector placed at $r$, with surface area $\Delta S$ and normal $\hat{n}$. The detector of quasi-momentum has an elongated form and is directed along the vector $\hat{\eta}$.

The particular form of each factor corresponds to a given experimental condition. When the characteristic length of the source and the characteristic time of duration of generated pulses are the shortest characteristic quantities of the problem one can assume that $[1-3]$

$$
\dot{\zeta}(Q ; \boldsymbol{r}, t)=A(Q) \delta(r) \delta(t)
$$

where $\delta(x)$ is the Dirac delta function, $\delta(r)=\delta(x) \delta(y) \delta(z)$.

When the phonon wave packets are generated by heating a metallic film, one can assume that [3]

$$
F_{1}(r) \equiv F_{2 \mathrm{D}}^{(\mathrm{G})}(r)=\delta(z)\left(2 \pi r_{\mathrm{eff}}^{2}\right)^{-1} \exp \left[\frac{-r_{\perp}^{2}}{2 r_{\mathrm{eff}}^{2}}\right]
$$

where $r_{\text {eff }}$ is a length characterizing the linear dimension of the heated area and $r_{\perp}$ is the component of $r$ perpendicular to the $z$ axis. The circular area $\pi r_{\text {eff }}^{2}$ can be interpreted as the effective area of the phonon source. which

In our previous papers $[1,2]$ we considered a monochromatic source, for

$$
A(Q)=\frac{4 \pi^{2} c^{3}(\hat{Q}) N_{\mathrm{ph}}}{3 \omega^{2}(Q)} \theta\left[v_{z}(\widehat{Q})\right] \delta\left[\omega(Q)-\omega_{0}\right]
$$

where $\theta(x)$ is the unit step function and $N_{\mathrm{ph}}$ is the number of phonons introduced into the specimen. Further we take $N_{\mathrm{ph}}=1$. The monochromatic source term obeys the normalization condition

$$
\int_{V_{+}} \mathrm{d}^{3} r \int_{-\infty}^{\infty} \mathrm{d} t \sum_{j=0}^{2} \frac{1}{(2 \pi)^{3}} \int \mathrm{d}^{3} q \dot{\zeta}_{\text {mon }}(Q ; r, t) \hbar \omega=\hbar \omega_{0}
$$


where $V_{+}$is defined by three inequalities

$$
-\infty \leq x \leq+\infty, \quad-\infty \leq y \leq+\infty, \quad-\varepsilon \leq z \leq+\infty,(\varepsilon \rightarrow 0) .
$$

IIere we shall consider also Planckian sources at temperature $T_{\mathrm{h}}$ higher than the ambient temperature $T$. Then, we will take the function $A(Q)$ to be of the following form

$$
A(Q) \equiv A_{\mathrm{pl}}\left(\omega, T_{\mathrm{h}}\right)=2 V_{\mathrm{h}} p N_{0}\left(\frac{\hbar \omega}{k_{\mathrm{B}} T}\right) \theta\left[v_{z}(\hat{Q})\right] .
$$

The explicit form of the coefficient $p$ is obtained from the normalization condition. We take it to be of the following form

$$
\int_{V_{+}} \mathrm{d}^{3} r \int_{-\infty}^{\infty} \mathrm{d} t \sum_{j=0}^{2} \frac{1}{(2 \pi)^{3}} \int \mathrm{d}^{3} q \dot{\zeta}_{\mathrm{pl}}(Q ; r, t) \hbar \omega=\alpha(\mathrm{h} \rightarrow \mathrm{s}) E_{\mathrm{th}}\left(T_{\mathrm{h}}\right) .
$$

From (21) it follows that

$$
p=\left[\frac{c_{\mathrm{D}}^{(\mathrm{s})}}{c_{\mathrm{D}}^{(\mathrm{h})}}\right]^{3} \alpha(\mathrm{h} \rightarrow \mathrm{s}) .
$$

If the time duration of the generated pulses $t_{\mathrm{p}}$ is finite (i.e. $t_{\mathrm{p}}$ is not the shortest time scale of the problem) one can assume that

$$
F_{2}=\exp \left(-\frac{t}{t_{\mathrm{p}}}\right)
$$

\section{Density of energy and quasi-momentum current}

The solution of Eq. (14) with the source term (16) is proportional to the Green function of the BE, which we denote $G(r, t)$

$$
\delta N_{p}(Q, r, t)=A(Q) G(r, t),
$$

where

$$
G(r, t)=\theta(t) \delta[r-v(\widehat{Q}) t] .
$$

The solution of the BE for an arbitrary production function $\delta N_{\dot{\zeta}}(Q ; r, t)$

$$
\dot{\zeta}(Q ; r, t)=A(Q) F_{1}(r) F_{2}(t)
$$

is

$$
\delta N_{\dot{\zeta}}(Q ; r, t)=\int \mathrm{d}^{3} r^{\prime} \int_{-\infty}^{\infty} \mathrm{d} t^{\prime} G\left(Q ; r-r^{\prime}, t-t^{\prime}\right) \dot{\zeta}\left(Q ; r^{\prime}, t^{\prime}\right) .
$$

Accounting for the Green function one can rewrite (25) in the form

$$
\delta N_{\dot{\zeta}}(Q ; r, t)=A(Q) \int_{-\infty}^{t} \mathrm{~d} t^{\prime} F_{1}\left[r-v(\hat{Q})\left(t-t^{\prime}\right)\right] F_{2}\left(t^{\prime}\right) .
$$

In particular for the Gaussian flat source producing infinitely short pulses one finds

$$
\delta N_{2 \mathrm{DG}}(Q ; r, t)=A(Q) \theta(t) \delta\left[z-v_{z}(\widehat{Q}) t\right] \exp \left[-\frac{\left(r_{\perp}-v_{\perp} t\right)^{2}}{2 r_{\mathrm{eff}}^{2}}\right]\left(2 \pi r_{\mathrm{eff}}^{2}\right)^{-1}
$$




\section{Densities of energy and quasi-momentum for different sources}

The explicit expressions for the phonon distribution function $\delta N(Q ; \boldsymbol{r}, t)$ allow us to calculate the components of the energy density current $j_{E}(r, t)$ and the tensor of the quasi-momentum density current $\pi(r, t)$ (cf. Refs. [8, 9])

$$
\begin{aligned}
& j_{E}^{\alpha}(r, t)=\sum_{j=0}^{2} \frac{1}{(2 \pi)^{3}} \int \mathrm{d}^{3} q \hbar \omega(Q) v_{\alpha}(\widehat{Q}) \delta N(Q ; r, t), \\
& \pi_{\alpha \beta}(r, t)=\sum_{j=0}^{2} \frac{1}{(2 \pi)^{3}} \int \mathrm{d}^{3} q \hbar q_{\alpha} v_{\beta}(\widehat{Q}) \delta N(Q ; r, t) .
\end{aligned}
$$

For a bolometer placed at $\boldsymbol{r}$ with surface of area $\Delta S$ and normal $\widehat{n}$, the total energy falling onto it is

$$
E(r)=\sum_{j=0}^{2} E_{j}(r)=\sum_{j=0}^{2} \int_{0}^{\infty} \mathrm{d} t j_{E}(r, t) \widehat{n} \Delta S,
$$

while for a quasi-momentum detector directed along $\widehat{\eta}$

$$
P_{\eta}(r)=\sum_{j=0}^{2} P_{\eta}^{(j)}(r)=\sum_{j=0}^{2} \int_{0}^{\infty} \mathrm{d} t \widehat{\eta}_{\alpha} \pi_{\alpha \beta}(r, t) \widehat{n}_{\beta} \Delta S .
$$

In fact we calculate the suitable densities

$$
\begin{aligned}
& e(r)=\frac{E(r)}{\Delta S}, \\
& p_{\eta}(r)=\frac{P_{\eta}(r)}{\Delta S} .
\end{aligned}
$$

Let us consider the densities $e(r, t)$ and $p(r, t)$ for point sources. For this purpose we shall use a formula relating the Dirac delta function $\delta\left(r-v_{j} t\right)$ to the focusing factor $\mathcal{A}_{j}(\theta, \phi)$ obtained in I, namely

$$
\begin{aligned}
& \delta(r-\left.v_{j} t\right)=\frac{1}{v_{j} r^{2}\left|\sin \theta_{v}\right|} \delta\left(t-\frac{r}{v_{j}}\right) \\
& \quad \times \sum_{i=1}^{n_{j}} \delta\left[\theta-\theta_{j}^{(i)}\right] \delta\left[\phi-\phi_{j}^{(i)}\right] \mathcal{A}_{j}(\theta, \phi)\left|\frac{\sin \theta_{v}^{(j)}}{\sin \theta}\right|,
\end{aligned}
$$

where the angles $\theta_{j}^{(i)}, \phi_{j}^{(i)}(i=1,2, \ldots, n)$ are the solutions of the three pairs of equations

$$
\theta_{v}^{(j)}(\theta, \phi)=\theta_{r}, \quad \phi_{v}^{(j)}(\theta, \phi)=\phi_{r} \quad(j=0,1,2) .
$$

For an arbitrary function $F_{j}$ of $\theta$ and $\phi$ we denote

$$
F_{j}^{(i)}\left(\theta_{r}, \phi_{r}\right)=F_{j}\left[\theta_{j}^{(i)}\left(\theta_{r}, \phi_{r}\right), \phi_{j}^{(i)}\left(\theta_{r}, \phi_{r}\right)\right] \quad(j=0,1,2) .
$$

We are now ready to write down the results for point monochromatic and Planckian sources. For a monochromatic point source we find (cf. I)

$$
e(\boldsymbol{r})=\frac{(\widehat{n} \cdot \widehat{r})}{2 \pi r^{2}} \frac{1}{3} \sum_{j=0}^{2} \sum_{i=1}^{n_{j}} \mathcal{A}_{j}^{(i)}\left(\theta_{r}, \phi_{r}\right) \hbar \omega_{0},
$$




$$
p_{\eta}(r)=\frac{(\widehat{n} \cdot \widehat{r})}{2 \pi r^{2}} \frac{1}{3} \sum_{i=1}^{n_{j}}\left[\widehat{\eta} s_{j}^{(i)}\left(\theta_{r}, \phi_{r}\right)\right] \mathcal{A}_{j}^{(i)}\left(\theta_{r}, \phi_{r}\right) \hbar \omega_{0} .
$$

For a Planckian point source we obtain similar expressions

$$
\begin{aligned}
e(r)= & \frac{E_{\mathrm{th}} \alpha(\mathrm{h} \rightarrow \mathrm{s})(\widehat{n} \cdot \widehat{r})}{2 \pi r^{2}} \frac{1}{3} \sum_{j=0}^{2} \sum_{i=1}^{n_{j}} \mathcal{A}_{j}^{(i)}\left(\theta_{r}, \phi_{r}\right)\left[\frac{c_{\mathrm{D}}^{(\mathrm{s})}}{c_{j}^{(i)}\left(\theta_{r}, \phi_{r}\right)}\right]^{3}, \\
p_{\eta}(r)= & \frac{E_{\mathrm{th}} \alpha(\mathrm{h} \rightarrow \mathrm{s})(\widehat{n} \cdot \widehat{r})}{2 \pi r^{2}} \frac{1}{3} \sum_{j=0}^{2} \sum_{i=1}^{n_{j}} \mathcal{A}_{j}^{(i)}\left(\theta_{r}, \phi_{r}\right) \\
& \times\left[\widehat{\eta} s_{j}^{(i)}\left(\theta_{r}, \phi_{r}\right)\right]\left[\frac{c_{\mathrm{D}}^{(\mathrm{s})}}{c_{j}^{(i)}\left(\theta_{r}, \phi_{r}\right)}\right]^{3} .
\end{aligned}
$$

Note that both densities $e(r), p(r)$ are proportional to the fourth power of the source temperature $T_{\mathrm{h}}$. This theoretical prediction can be experimentally verified.

For a Gaussian source the focusing factor is changed by a Gaussian exponential function. Namely, for a monochromatic source we obtain

$$
\begin{aligned}
e(r) & =\frac{\hbar \omega_{0}}{2 \pi r_{\mathrm{eff}}^{2}} \frac{1}{3} \sum_{j=0}^{2} \int_{4 \pi} \frac{\mathrm{d} \widehat{q}}{2 \pi} \theta\left[v_{z}(\widehat{Q})\right] \frac{[\widehat{n} \cdot v(\widehat{Q})]}{v_{z}(\widehat{Q})} \\
& \times \exp \left\{-\left[r_{\perp}-\frac{v_{\perp}(\widehat{Q}) z}{v_{z}(\widehat{Q})}\right]^{2}\left(2 \pi r_{\mathrm{eff}}^{2}\right)^{-1}\right\}, \\
p_{\eta}(r) & =\frac{\hbar \omega_{0}}{2 \pi r_{\mathrm{eff}}^{2}} \frac{1}{3} \sum_{j=0}^{2} \int_{4 \pi} \frac{d \widehat{q}}{2 \pi} \theta\left[v_{z}(\widehat{Q})\right] \frac{[\widehat{n} \cdot v(\widehat{Q})]}{v_{z}(\widehat{Q})}[\widehat{\eta} s(\widehat{Q})] \\
& \times \exp \left\{-\left[r_{\perp}-\frac{v_{\perp}(\widehat{Q}) z}{v_{z}(\widehat{Q})}\right]^{2}\left(2 \pi r_{\mathrm{eff}}^{2}\right)^{-1}\right\} .
\end{aligned}
$$

Finally we list the results for a Gaussian source of Planckian heat pulses

$$
\begin{aligned}
e(r) & =\frac{E_{\mathrm{th}} \alpha(\mathrm{h} \rightarrow \mathrm{s})}{2 \pi r_{\mathrm{eff}}^{2}} \frac{1}{3} \sum_{j=0}^{2} \int_{4 \pi} \frac{\mathrm{d} \widehat{q}}{2 \pi} \theta\left[v_{z}(\widehat{Q})\right] \frac{[\widehat{n} \cdot v(\widehat{Q})]}{v_{z}(\widehat{Q})}\left[\frac{c_{\mathrm{D}}^{(\mathrm{s})}}{c(\widehat{Q})}\right]^{3} \\
& \times \exp \left\{-\left[r_{\perp}-\frac{v_{\perp}(\widehat{Q}) z}{v_{z}(\widehat{Q})}\right]^{2}\left(2 \pi r_{\mathrm{eff}}^{2}\right)^{-1}\right\} \\
p_{\eta}(r) & =\frac{E_{\mathrm{th}} \alpha(\mathrm{h} \rightarrow \mathrm{s})}{2 \pi r_{\mathrm{eff}}^{2}} \frac{1}{3} \sum_{j=0}^{2} \int_{4 \pi} \frac{\mathrm{d} \widehat{q}}{2 \pi} \theta\left[v_{z}(\widehat{Q})\right] \frac{[\widehat{n} \cdot v(\widehat{Q})}{v_{z}(\widehat{Q})}\left[\frac{c_{\mathrm{D}}^{(\mathrm{s})}}{c(\widehat{Q})}\right]^{3}[\widehat{\eta} s(\widehat{Q})] \\
& \times \exp \left\{-\left[r_{\perp}-\frac{v_{\perp}(\widehat{Q}) z}{v_{z}(\widehat{Q})}\right]^{2}\left(2 \pi r_{\mathrm{eff}}^{2}\right)^{-1}\right\} .
\end{aligned}
$$

Equations (38)-(45) are used for calculation of the focusing patterns. 


\section{Method of numerical calculation}

Until now our analytical calculations have been performed for point detectors [1, 2]. Nevertheless, in real experiments detectors have finite linear dimensions. Usually in numerical calculations even finite size detectors are treated as a superposition of point detectors. However, in the case of an extremely inhomogenous distribution of phonons such an approximation is not acceptable, since the sums calculated during the numerical calculations over a finite size detector area inevitably contain infinitely large terms. This happens when the focusing coefficient (defined as the limit of the absolute value of the ratio (5) for $\mathrm{d} \Omega_{q} \rightarrow 0$ ) diverges, i.e. in the presence of caustics. We note that the calculation of the map of the phonon arrival time is still possible.

The following approach allows us to overcome the noted difficulty. Consider the body angle $\Omega_{q}$ subtencied by a bundle of three vectors $q_{1}, q_{2}, q_{3}$. For each of these wave vectors and for a chosen polarization $j$ one can calculate the corresponding vectors of the group velocity $v\left(q_{1}, j\right), v\left(q_{2}, j\right), v\left(q_{3}, j\right)$. With the bundle of group velocity vectors there is associated a body angle $\Omega_{v}^{j}$. The detector surface is seen from a point source under the body angle $\Omega_{d}$. Denote the common part of the body angles $\Omega_{v}^{j}$ and $\Omega_{d}$ by $\Omega_{c}^{j}$, i.e.

$$
\Omega_{c}^{j}=\Omega_{v}^{j} \cap \Omega_{d}, \quad(j=0,1,2) .
$$

Denote the energy transported by phonons of polarization $j$ with the mean wave vector contained in $\Omega_{q}$ by $E_{\Omega}^{(j)}$. The contribution of these phonons to the energy falling onto the detector is

$$
E_{\Omega q}^{(j)} \Omega q \frac{\Omega_{c}^{j}}{\Omega_{v}^{j}}
$$

Summing over all body angles $\Omega_{\boldsymbol{q}}$ in the reciprocal space (and eventually over all three polarizations $j$ ) one can obtain a partial energy $E_{j}$ or the total energy $E_{\text {tot }}$ falling onto the detector surface, e.g.

$$
E_{\mathrm{tot}}=\sum_{j=0}^{3} E_{j}=\sum_{j=0}^{3} \sum_{\Omega \boldsymbol{q}} E_{\Omega}^{(j)} \Omega \boldsymbol{q} \frac{\Omega_{c}^{j}}{\Omega_{v}^{j}} .
$$

Note that the sum defining $E_{\text {tot }}$ does not contain infinite terms since all body angles $\Omega_{\boldsymbol{q}}$ are finite and the last factor on the right hand sides of (46)-(47) obeys the obvious condition

$$
0 \leq \frac{\Omega_{c}^{j}}{\Omega_{v}^{j}} \leq 1
$$

Besides, the ratio $\Omega_{q} / \Omega_{v}^{j}$, defining the focusing coefficient in the limit $\Omega_{q} \rightarrow 0$, is also present in formula (47).

Until now we considered experiments with a fixed detector and movable source. However several experiments were performed with a fixed source and a movable detector (cf. $[10,11,12])$. Both configurations are completely equivalent. So we assume that the detector is movable and the source fixed.

Next we shall fix the grid of possible detector positions and scan the reciprocal space with small body angles $\Omega_{q_{i}}$ formed by bundles of triples of non 
coplanar unit vectors $q_{1 i}, q_{2 i}, q_{3 i}(i=1,2,3, \ldots, m ; m \gg 1)$. As we assumed that for $(i \neq l)$ the body angles $\Omega_{q_{i}}, \Omega_{q_{i}}$ do not overlap

$$
\Omega_{\boldsymbol{q}_{\mathrm{i}}} \cap \Omega_{\boldsymbol{q}_{\mathrm{i}}}=0 \quad(i \neq l)
$$

hence

$$
\bigcup_{i=1}^{m} \Omega_{\boldsymbol{q}_{i}}=4 \pi
$$

For a given polarization index $j(j=0,1,2)$ we calculate simultaneously the contributions (46) from the body angle $\Omega_{\boldsymbol{q}_{i}}$ to all detectors for which $\Omega_{\mathrm{c}}^{j} \neq 0$. In this way we achieve a great reduction of the amount of calculation. Further, a reduction of the number of calculations is achieved by accounting for the symmetry of the crystalline material and the sample. The accuracy of the calculations depends mainly on the number $m$.

The present method may easily be generalized to the calculation of quasi-momentum focusing patterns [1] or the phonon induced drag current in a 2D electron gas [2]. Simply in the first case instead of the energy $E_{\Omega q}^{(j)}$ a suitable quantity for the mean vector contained in $\Omega_{q}$ should be calculated and, in the second case, the suitable quantity should be calculated according to the rule (47).

\section{Results of numerical calculation}

The angular distribution of energy for a monochromatic source is determined by the focusing factor $\mathcal{A}_{j}^{(i)}\left(\theta_{r}, \phi_{r}\right)$ (cf. Eqs. $(38,39)$ ) while for a Planckian source this term is replaced by

$$
\mathcal{A}_{j}^{(i)}\left(\theta_{r}, \phi_{r}\right)\left[\frac{c_{\mathrm{D}}^{(\mathrm{s})}}{c_{j}^{(i)}\left(\theta_{r}, \phi_{r}\right)}\right]^{3}
$$

(cf. Eqs. $(40,41)$ ). Thus one expects noticeable differences between the corresponding patterns only for media with strongly anisotropic phase velocities. We assumed that the linear dimension $r_{\mathrm{D}}$ of the detector is small (it is "point-like"). In the calculation we take $r_{\mathrm{D}}=7.8 \mu \mathrm{m}$. Comparing the energy focusing patterns for point monochromatic (Fig. 2a) and point Planckian (Fig. 3a) sources of phonons we conclude that the visible difference is rather minor and probably beyond the reach of experimental accuracy.

According to formulae (42-45), the phonon images obtained for Gaussian sources depend on the quotient $r_{\text {eff }} / d$, where $d$ is the sample thickness (i.e. $z=d$ ). For $r_{\text {eff }} / d \ll 1$ the patterns produced by point sources do not differ substantially from those given by Gausssian sources. In the other case, the caustics of the patterns corresponding to Gaussian sources become smeared out. This effect is shown in Figs. 2b,c (for a monochromatic source) and $3 b, c$ (for a Planckian source). For a given thickness $d$ and increasing $r_{\mathrm{eff}}$, the patterns for monochromatic sources and for Planckian sources become noticeably different. In principle, for a given type of source (monochromatic or Planckian) the comparison of the experimentally obtained patterns with the calculated ones allows us to estimate the effective 

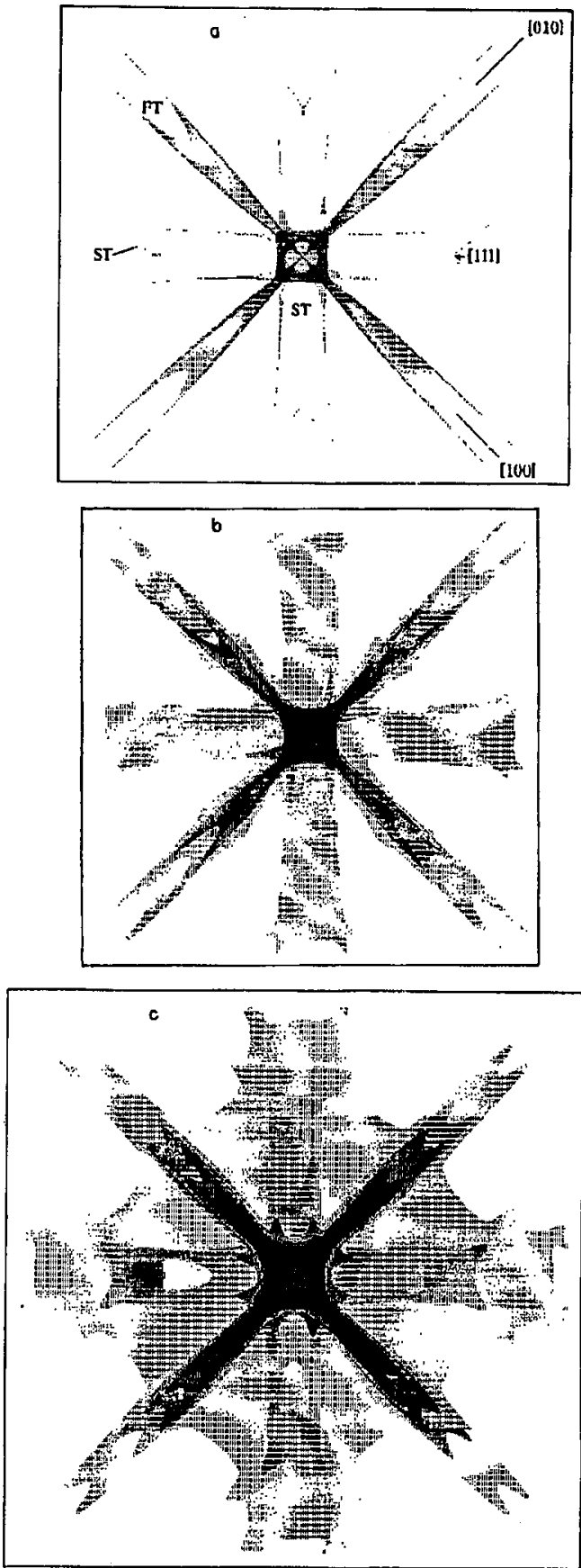

Fig. 2. (a) The calculated energy focusing pattern for a point monochromatic source on a $\{001\}$ plane of GaAs, all three acoustic phonon modes. (b), (c) The same as in Fig. 2 a for Gaussian sources of monochromatic phonons: (b) $r_{\mathrm{eff}}=31 \mu \mathrm{m}$, (c) $r_{\mathrm{eff}}=62 \mu \mathrm{m}$. 

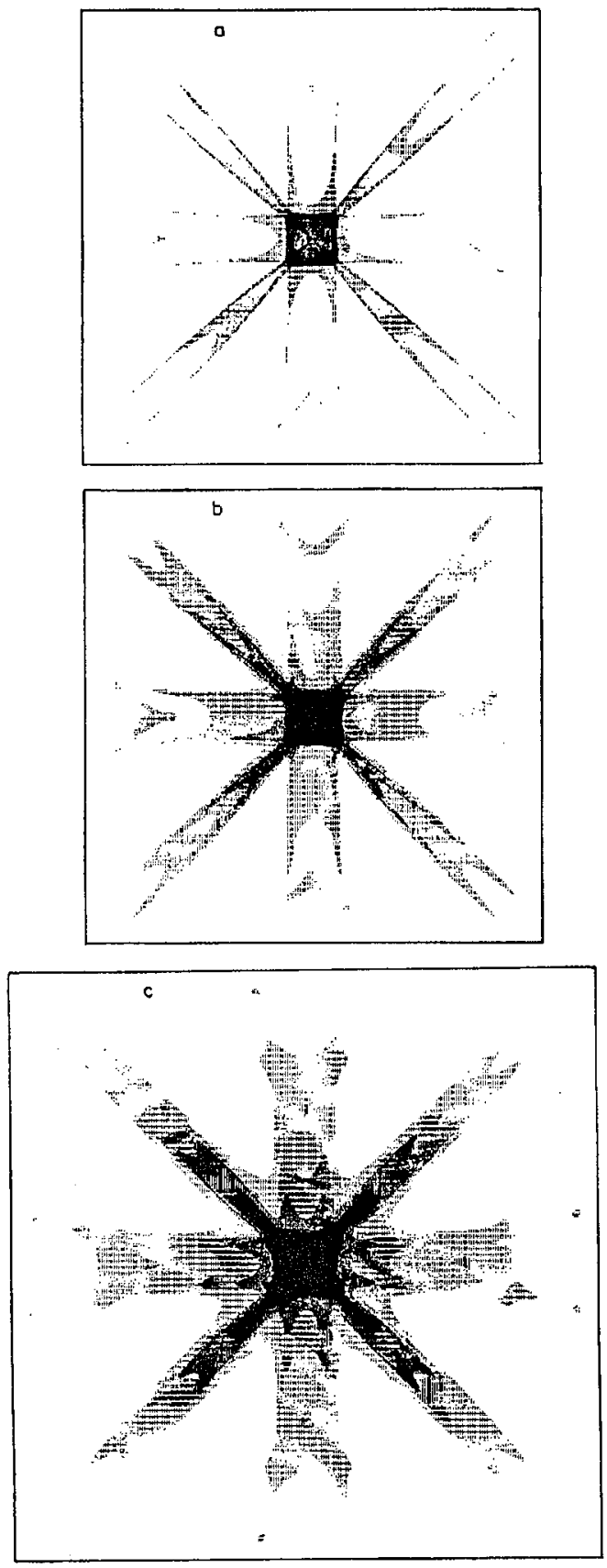

Fig. 3. (a) The calculated energy focusing pattern for a point Planckian source on a \{001\} plane of GaAs, all three acoustic phonon modes. (b), (c) The same as in Fig. 3a for Gaussian sources of Planckian phonons: (b) $r_{\mathrm{eff}}=31 \mu \mathrm{m}$, (c) $r_{\mathrm{eff}}=62 \mu \mathrm{m}$. 

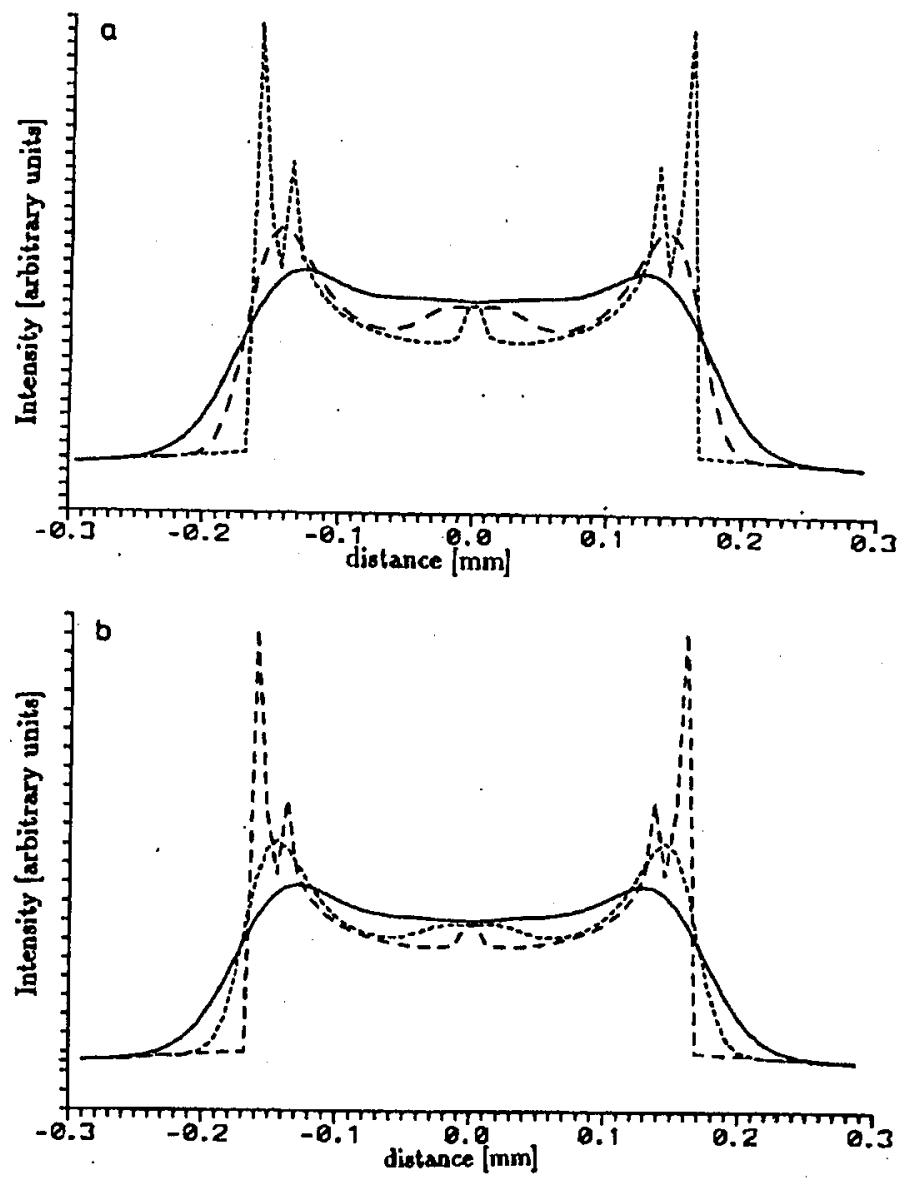

Fig. 4. (a) Scans of the energy density for monochromatic extended (Gaussian) source along the horizontal line crossing the pattern centre. Long dashed lines $-r_{\text {eff }}=0$, short dashed lines $-r_{\mathrm{eff}}=31 \mu \mathrm{m}$, solid lines $-r_{\mathrm{eff}}=62 \mu \mathrm{m}$. (b) Scans of the energy density for Planckian extended (Gaussian) source along the horizontal line crossing the pattern centre. Long dashed lines $-r_{\mathrm{eff}}=0$, short dashed lines $-r_{\mathrm{eff}}=31 \mu \mathrm{m}$, solid lines $r_{\text {eff }}=62 \mu \mathrm{m}$.

width $r_{\text {eff }}$ of the source. Knowing the size of the source we can estimate the local temperature rise $\left(T_{\mathrm{h}}\right)$ caused by a pulsed beam of radiation or particles. It seems that this will provide a valuable test of the existing theories of the heating of normal and superconducting films or surfaces of dielectrics and semiconductors by such beams.

The better qualitative analysis of the obtained results is achieved upon comparing line scans of the energy density as shown in Fig: 4a (monochromatic source) and Fig. $4 \mathrm{~b}$ (Planckian source). It is assumed that the characteristic length of the detector is $7.8 \mu \mathrm{m}$. The experimentaly obtained scan [5] is shown in Fig. 5 . 


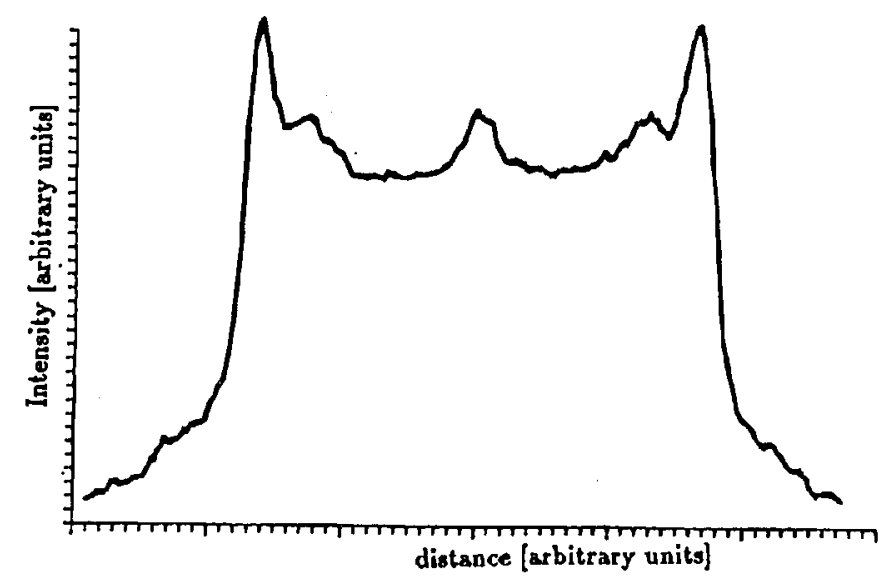

Fig. 5. The experimentally obtained scan along the horizontal line crossing the pattern centre. It is assumed that the source is Gaussian $\left(r_{\mathrm{eff}}=20 \mu \mathrm{m}\right)$ and its effective temperature is $7.8 \mathrm{~K}[5]$.

If the image does not contain caustics it is not sensitive to the ratio $r_{\mathrm{eff}} / d$. This feature is demonstrated in Fig. 4.

\section{Concluding remarks}

We have shown that for pulses of monochromatic and Planckian sources of phonons, the distribution of the energy falling onto one of crystallographic planes depends upon the source radius $r_{\text {eff. }}$. In principle comparison of the calculated focusing patterns allows us to discriminate between the monochromatic and Planckian sources and to estimate the source radius $r_{\text {eff }}$.

Usually detectors of phonon energy are superconducting films. Consequently, we identified the energy density with the response of such films to pulsed phonon beams. However, in the case of phonon quasi-momentum detectors (low dimensional gases of carriers) the quasi-momentum focusing pattern and the detector response pattern differ essentially. Therefore we calculated also the quasi-momentum density and the phonon induced drag current density patterns for low dimensional gases of carriers (1D and 2D gases of electrons and holes). Preliminary results are reported in this issue.

\section{Acknowledgments}

This work is supported by a grant from the Committee for Scientific Research under the contract No. $397 / 2 / 91$.

\section{References}

[1] Cz. Jasiukiewicz, D. Lehmann, T. Paszkiewicz, Z. Phys. B, Condens. Matter 84, 73 (1991). 
[2] Cz. Jasiukiewicz, D. Lehmann, T. Paszkiewicz, Z. Phys. B, Condens. Matter 86, 225 (1992).

[3] Cz. Jasiukiewicz, T. Paszkiewicz, in: Phonon Scattering in Condensed Matter, Eds. M. Meissner, R.O. Pohl, Springer, Berlin, Heidelberg, New York 1993, p. 96.

[4] W. Metzger, R.P. Huebener, Z. Phys. B, Condens. Matter 73, 33 (1988).

[5] E. Held, W. Klein, R.P. Huebener, Z. Phys. B, Condens. Matter 75, 17 (1989).

[6] F. Dietzel, R. Wichard, W. Beckes, W. Dietsche, K. Ploog, Verh. Dtsch. Phys. Ges. 8, 1065 (1991).

[7] E.T. Swartz, R.O. Pohl, Rev. Mod. Phys. 61, 605 (1989).

[8] J.A. Kobussen, T. Paszkiewicz, Helv. Phys. Acta 54, 383 (1981).

[9] J.A. Kobussen, T. Paszkiewicz, Helv. Phys. Acta 54, 396 (1981).

[10] A.V. Akimov, A.A. Kaplyanskii, E.S. Moskalenko, R.A. Titov, Zh. Exp. Theor. Fiz. 94, 307 (1988).

[11] L.J. Challis, A.J. Kent, V.W. Rampton, in: Phonons 89, Eds. S. Hunklinger, W. Ludwig, G. Weiss, World Scientific, Singapore 1990, p. 967.

[12] L.J. Challis, A.J. Kent, in: Proc. 29th Winter School of Theoretical Physics, Eds. T. Paszkiewicz, K. Rapcewicz, Plenum Press, New York 1993, in preparation. 